\title{
BMJ Open Prevalence and diagnostic value of GPs' gut feelings for cancer and serious diseases: protocol for a prospective observational study of diagnostic validity
}

\author{
Bernardino Oliva-Fanlo (D) ,1,2 Sebastià March, ${ }^{1,3,4}$ David Medina, ${ }^{1,3,4,5}$ \\ María Martín-Rabadán, 1,3,4,6 Gaspar Tamborero, ${ }^{1,7}$ Erik Stolper, 8,9 \\ Magdalena Esteva ${ }^{1,3,4}$
}

To cite: Oliva-Fanlo B, March S, Medina D, et al. Prevalence and diagnostic value of GPs' gut feelings for cancer and serious diseases: protocol for a prospective observational study of diagnostic validity. BMJ Open 2019;9:e032404. doi:10.1136/ bmjopen-2019-032404

- Prepublication history for this paper is available online. To view these files, please visit the journal online (http://dx.doi. org/10.1136/bmjopen-2019032404).

Received 17 June 2019 Revised 15 September 2019 Accepted 17 September 2019

Check for updates

(c) Author(s) (or their employer(s)) 2019. Re-use permitted under CC BY-NC. No commercial re-use. See rights and permissions. Published by BMJ.

For numbered affiliations see end of article.

Correspondence to Dr Bernardino Oliva-Fanlo; boliva@ibsalut.caib.es

\section{ABSTRACT}

Introduction Cancer diagnosis in primary care is an important challenge for general practitioners (GPs) due to the relatively low frequency of any single type of cancer and the heterogeneous signs and symptoms that can be present. In addition to analytical reasoning, GPs may become aware of gut feelings (GFs) as they suspect that a patient may have cancer or another serious disease. We aimed to investigate the prevalence and the predictive value of GFs for the diagnosis of cancer and serious diseases.

Methods and analysis Prospective observational study of diagnostic validity. Participation will be offered to GPS from Majorca and Zaragoza (Spain). They will recruit all patients with a new reason for encounter during one or two workdays. GPs will complete the Gut Feelings Questionnaire (GFQ). Variables regarding patient, GP and consultation will be collected. Two and 6 months after the first visit, incident diagnoses of cancer or other serious diseases, diagnostic tests performed, referrals and new visits will be recorded. Analysis will include a descriptive analysis of the variables and prevalence of GFs, and the sensitivity, specificity, predictive values and likelihood ratios of the GFs (sense of alarm and sense of reassurance) for diagnosing cancer and other serious diseases, as measured with the GFQ.

Ethics and dissemination The study has obtained approval from the Majorcan Primary Care Research Committee and from the Balearic Islands Ethical Committee, with reference number IB 3210/16 PI. The results may help GPs to make more accurate decisions about which patients need further examinations to rule out or to confirm a diagnosis of cancer or a serious disease, and which ones do not. The results will be published as part of the PhD project of the first author and in open access journals, and will be presented at medical conferences.

\section{INTRODUCTION}

Establishing a diagnosis of cancer in primary care presents many difficulties. A cancer

\section{Strengths and limitations of this study}

This is the first study to use an objective tool (the Gut Feeling Questionnaire) to assess the prevalence and the diagnostic value of gut feelings (GFs) for the diagnosis of cancer and other serious diseases.

- We will analyse variables that can affect the prevalence and diagnostic value of GFs.

- The study will focus on both types of GF: the sense of alarm and the sense of reassurance.

- The results of this study may help to estimate the extent to which GPs' GFs can contribute to the diagnosis of cancer and serious diseases.

- The results of the study might be influenced by the Hawthorne effect.

diagnosis is not a rare event since a general practitioner (GP) with 2000 registered patients will see six to eight new cases per year. However, on average, a GP will diagnose a case of each of the most common cancers (colorectal, prostate, breast and lung) only once a year, and less frequent cancers might be seen only once or twice during a GP's career. ${ }^{1}$ In addition, signs and symptoms of cancer are different for each cancer type, and these signs and symptoms are also very common in other, mostly mild, diseases. Even those considered to be alarming symptoms have low positive predictive values (PPVs) for cancer diagnosis; only eight signs and symptoms (rectal bleeding, iron deficiency anaemia, haematuria, rectal examination showing malignancy, haemoptysis, dysphagia, breast lump and postmenopausal bleeding) have a PPV above $5 \%{ }^{2}$ Since half of patients with cancer do not have alarming symptoms, ${ }^{3}$ there is a growing interest in finding new elements in the diagnostic process that would 
lead to an earlier diagnosis in primary care with better survival results.

Uncertainty is an intrinsic component of any clinical encounter in general practice. To cope with it, GPs not only use analytical processes but sometimes also rely on what they call a gut feeling (GF) or an intuition. ${ }^{4}$ GFs have been described as 'a useful warning light that goes on suddenly to announce that there is something unusual ${ }^{5}$ It is in the grey area of common symptoms and vague signs, where uncertainty dominates, that GPs might gain the greatest benefit from becoming aware of GFs. When they do so, they have to ask more specific questions, adopt an even more attentive attitude and observe more accurately to identify the trigger that gave them the sense of alarm. The role of GFs in the GP's diagnostic process has been studied in countries such as the Netherlands, Belgium, France and Spain. ${ }^{6-9}$ These studies show two types of GF. The first one is a sense of alarm, described as the feeling that something does not fit for a particular patient, making the GP worried about a possible serious outcome. The second type of GF consists of a sense of reassurance, in which the doctor is sure about the future evolution and management of the patient, even if he/ she does not yet know the precise diagnosis. The majority of studies on GFs carried out so far have used qualitative methodology. As a result of the findings, Dutch and Belgian researchers have created and validated the Gut Feelings Questionnaire (GFQ) to objectify their occurrence in clinical encounters. ${ }^{10}$ The Dutch GFQ has been translated and linguistically validated into English, French, German, Polish, Spanish and Catalan. ${ }^{11} 12$

\section{Limitations of existing literature}

The sense of alarm arises especially when diffuse symptoms are suspected to hide a neoplasm. ${ }^{679}$ The role of GFs in the diagnosis of cancer has been little studied so far. In an English study, 55 GPs were interviewed about the role of GFs in the screening and early diagnosis of cancer. $^{13}$ They referred to GFs as a tool, developed through experience, useful to identify patients who need more complementary tests, either to confirm or to rule out the possible presence of cancer. Norwegian GPs were asked how they came to think of cancer in a clinical encounter. ${ }^{4}$ They mentioned intuition and GFs as one of the ways in which that idea arises. They described GFs as resulting from their medical knowledge, accumulated expertise and knowledge about the patient and their community. There have also been two Danish studies using a quantitative approach to determine the accuracy with which GPs diagnose cancer. In one of them, 4518 consultations were studied..$^{14}$ After each consultation, the GP answered the question whether he had any suspicion of cancer or serious illness. The suspicion of cancer had a PPV of $3.1 \%$ and a negative predictive value (NPV) of $99.5 \% 6$ months after the consultation. The other study examined the reasons for referral for further diagnostic workup in a cancer pathway among 1218 patients with nonspecific symptoms and signs. ${ }^{15}$ GPs' GFs were the second most common reason for referral $(22.5 \%$ of cases), and a cancer diagnosis was established in $24 \%$ of these cases. Dutch GPs reported GFs relating to the diagnosis of cancer in 20 out of 10000 registered patients a year. These GFs were more likely to arise for patients with weight loss and patients who visited their GP infrequently. The predictive value of GFs for cancer diagnosis was $35 \%$, a value that increased with patients' or GPs' age. ${ }^{16}$

We aimed to study the diagnostic value of the GFQ for the diagnosis of cancer. Some studies have suggested that this diagnostic validity may be comparable to that of the recognised cancer alarm symptoms. ${ }^{2} 17$ If these results were confirmed, a GP's sense of alarm about possible cancer could be regarded as another alarm symptom or 'red flag'. GFs could be incorporated into the clinical training about the diagnostic process for medical students and residents. In addition, we will also address the sense of reassurance. Just like the GPs reported in previous qualitative literature, we think that it could be a very useful tool for doctors and patients to avoid unnecessary tests that could result in overmedicalisation and overdiagnosis.

\section{OBJECTIVES}

The aim of this study was to assess the diagnostic value of GPs' sense of alarm and sense of reassurance (in terms of sensitivity, specificity, predictive values and likelihood ratios) for cancer and other serious diseases in clinical consultations. We will also assess the relationships, if any, between the diagnostic value of GFs and patient characteristics (sociodemographic and clinical), and GP characteristics (gender, experience, personality, type of practice and knowledge about the patient) will also be assessed. A secondary objective was to establish the prevalence of GFs in GPs' consultations, as well as the possible relationship between the prevalence of GFs and patient and GP characteristics. We aimed to estimate the relationship between GFs (sense of alarm and sense of reassurance) and requests for tests and investigations, as well as referrals to hospital specialists.

\section{METHODS AND ANALYSIS}

This is a prospective observational study using the Spanish and Catalan versions of the GFQ.

\section{Participants}

GPs from the regions of Majorca and Zaragoza sector 1 (Spain) will be invited to participate. In Spain, every GP has his/her own patient lists. Patients are mainly attended by their GP at their health centre, except during holidays, sick leaves or in out-of-hours visits.

\section{GP inclusion}

A member of the researcher team will introduce the study in Majorca and Zaragoza health centres, inviting doctors to participate in the study. We will include similar 
proportions of teaching and non-teaching centres, as well as rural and urban centres. Those doctors who agree to participate will sign an informed consent form and receive a unique identification code. They will receive instructions about how to record the variables used in the study and how to complete the GFQ.

\section{Patient inclusion}

Consecutive patients consulting their GPs for at least one new reason during a working day will be included. A new reason for consultation is defined as the first time a particular patient consults the GP for this reason. For those patients with pathologies that present in repeated episodes over time (eg, acute low back pain), a new episode will be regarded as a new reason for consultation. Recurrence of cancer in cancer survivors who were considered disease-free after cancer treatment will also be regarded as a new diagnosis.

Scheduled visits, non-scheduled visits, home visits and telephone contacts will be included. At the end of the consultation, GPs will hand over an information sheet to each patient with a full explanation of the objectives and procedures of the study. If they agree to participate, they will sign an informed consent form.

Exclusion criteria include

- Consultations for administrative reasons (sick leaves, prescription renewals and reports).

- Patients in a terminal situation.

- Minors (under 18 years of age).

\section{Sample size}

Our sample size estimation was based on the Hjertholm study, ${ }^{14}$ which found the prevalence and PPV of cancer suspicion in GPs' consultations to be $6.0 \%$ and $5.4 \%$, respectively. Using an intraclass correlation coefficient correction and a proportion of losses of $10 \%$, we will need a sample of 2966 patients.

The Majorca and Zaragoza health sectors include a combined population of approximately 970000 inhabitants, 60 health centres and $500 \mathrm{GPs}$. We expect to recruit 150 GPs, who will be encouraged to include at least 20 patients with new reasons for encounter. We estimate that 8-10 patients per GP working day will consult with a new reason for encounter, so each GP will need to include patients during two to three working days in order to achieve the intended sample size.

\section{Data collection}

During consultations, the GPs will check inclusion and exclusion criteria and obtain informed consent. They will record demographic and clinical data and complete the GFQ using a printed version. The actual study will start after a pilot to assess the feasibility.

\section{Follow-up}

Two data managers will record the follow-up variables and outcomes. The presence of new diagnoses of cancer or serious disease will be recorded 2 and 6 months after the consultations. New diagnoses will be searched using diagnostic codes and free text notes. The incidence of cancer and serious disease will be assessed by reviewing the hospital and primary care electronic clinical records.

\section{Measurements}

Outcomes (follow-up variables)

After 2 and 6 months after the index visit, outcomes will include

- New diagnoses of cancer and serious diseases:

- Cancer: all new diagnoses, except non-melanoma skin cancer.

- Serious diseases: based on the list of diseases used by Hjertholm et al, ${ }^{14}$ all new diagnoses will be assessed by two researchers to decide if they are serious or not. Disagreements will be discussed and presented to a third researcher until agreement is reached.

- All diagnostic tests performed during the follow-up period. Referrals to specialised care and emergency departments.

- Numbers of consultations for any reason during the follow-up period.

\section{Independent variables}

\section{For the GP}

- Age, gender, native language, whether the GP is a trainer or not, rural/urban health centre, years of professional experience and years of caring for the same list of patients.

- GP's practice style (biomedical vs psychosocial) measured with a four-item scale validated in Spanish by Martínez-Cañavate. ${ }^{18}$

- Rational-Experiential Inventory (REI) scale, ${ }^{19}$ validated in Danish and Spanish populations ${ }^{20}$ to assess reasoning styles (rational or intuitive). The REI scale has two dimensions: rational and experiential. Each dimension contains 20 items and uses a 5-point Likert scale.

\section{For the patient}

- Sociodemographic (age, gender, country of origin and native language).

- Previously known to the GP? Since when?

\section{For the contact/visit}

- Type of consultation (scheduled or non-scheduled), home visits and phone calls.

- Language used.

- Date and time.

- Symptoms and signs: GPs will check if any symptom or sign included in a list of cancer-associated symptoms and signs is revealed in the consultation. This list has been adapted, using those with higher predictive values. ${ }^{2}{ }^{17}$ It includes loss of weight, anaemia, anorexia, asthenia, changes in bowel habits (diarrhoea and constipation), persistent dyspepsia, dysphagia, cough and dysphonia, lower urinary tract symptoms, unusual bleeding (haemoptysis, 
haematuria, rectal bleeding and vaginal postmenopausal), breast lump, abdominal mass and unusual pain.

- GFQ created and validated by Stolper et al..$^{10}$ The validated Spanish and Catalan versions will be used. ${ }^{12}$ The GFQ consists of 11 items. Item 1 (repeated at the end as item 11) assesses whether the patient's case elicited a GF. Items 2-6 are rated using a 5-point Likert scale ranging from completely disagree to completely agree. Item 2 concerns the sense of reassurance, and items 3-6 concern the sense of alarm. A sense of alarm is considered to be present when the answer to item 1 or 11 indicates a sense of alarm or when the answer chosen at item 1 or 11 is 'not applicable' and at least one of the scores of items $3-6$ is higher than $3 / 5$. A sense of reassurance is considered to be present when the answer to item 1 or 11 indicates a sense of reassurance or when the answer chosen at item 1 or 11 is not applicable and the score for item 2 is higher than $3 / 5$. No type of GF is considered to be present when the answer chosen at item 1 or 11 is not applicable, none of the scores for items $3-6$ is higher than $3 / 5$ and the score for item two is lower than $4 / 5$.

\section{Statistical analysis}

Objective 1

Sensitivity, specificity, PPVs and NPVs, and positive and negative likelihood ratios will be calculated for both sense of alarm and sense of reassurance. For the purpose of this calculation, we will assume that positive values for the sense of reassurance aim to identify the healthy patients, whereas positive values for the sense of alarm aim to identify ill patients. The outcome will be a new diagnosis of cancer, a cancer recurrence or a serious disease during the follow-up period. Information on a new diagnosis of cancer or cancer recurrences will be reported globally and specifically. CIs will be obtained for each parameter. Bivariate analysis will be used to explore the relationship between the sense of alarm and the sense of security and the patient and GP characteristics.

\section{Objective 2}

We will perform a descriptive analysis of all selected variables in order to describe sample characteristics and the prevalence of GFs.

\section{Objective 3}

A bivariate analysis will be carried out, in which each of the main variables (presence of sense of reassurance or sense of alarm) will be compared with the patient and GP characteristics. The $\chi^{2}$ test analysis will be used for categorical variables, and Student t-test or analysis of variance will be used for continuous variables. Multivariate logistic regression analysis will be used to assess the independent relationships between the variables and the presence of GFs. Interactions will be tested.

SPSS Statistics 23.0 software will be used for the analysis.

\section{Schedule}

All the documents (information sheet, informed consent forms and data collection sheets) have already been designed. Data will be collected during May, June and July 2019. Follow-up variables will be collected during August, September and October 2019 (2 months' follow-up), and December 2019 and January and February 2020 (6 months' follow-up).

\section{Limitations}

The sample size necessary to perform a diagnostic validity study of these characteristics is very large. The design we chose aimed to have a minimal impact on the consultations of the collaborating doctors, in order to facilitate their participation and the inclusion of patients. It is also a very adaptable design, since the number of recruitment days can be increased until the desired sample size is reached, although efforts will be made to include a large number of doctors to ensure sufficient practice variation. Selection bias is controlled by the consecutive inclusion of patients and by the instructions previously given to the collaborating physicians. To minimise the number of missing diagnostic records, the patient files of both primary care and hospital care will be thoroughly reviewed. In case of doubt, the seriousness of the diagnoses will be confirmed by peer review.

We are aware that the Hawthorne effect, that is, a change in a subject's behaviour due to the awareness of being studied, can be a source of bias. ${ }^{21}$ Participant GPs may thus perform differently than they would normally do, which may affect the estimation of the diagnostic value of GFs.

\section{Ethics and Dissemination}

The study has obtained approval from the Majorcan Primary Care Research Committee and the Balearic Islands Ethical Committee, with reference number IB 3210/16 PI.

The results of this study may help to estimate the extent to which GPs' GFs can contribute to the diagnosis of cancer and serious diseases. It will help GPs make more accurate decisions about which patients need further investigations and which ones do not.

This study is part of the corresponding author's $\mathrm{PhD}$ project, and its results will be published as part of the thesis and in open access journals, and presented at medical conferences.

\section{Author affiliations}

${ }^{1}$ Primary Care Research Unit of Mallorca, Balearic Health Services-IbSalut, Palma, Spain

${ }^{2}$ UBS Porreres (CS Ses Roques Llises), Majorca Primary Care Department, Porreres, Spain

${ }^{3}$ Can Misses Primary Health Centre, Balearic Islands Health Research Institute (IdISBa), Palma (I.Baleres), Spain

${ }^{4}$ Preventive Activities and Health Promotion Network, Carlos III Institute of Health (RedIAPP-RETICS), Madrid, Spain

${ }^{5}$ Manacor Primary Health Centre, Majorca Primary Care Department, Manacor, Spain 
${ }^{6}$ Can Misses Primary Health Centre, Ibiza and Formentera Health District, Eivissa, Spain

${ }^{7}$ Coll d'en Rabassa Primary Health Centre, Majorca Primary Care Department,

Palma, Spain

${ }^{8}$ Department of General Practice, Faculty of Health, Medicine and Life Sciences, Caphri School for Public Health and Primary Care, University of Maastricht, Maastricht, The Netherlands

${ }^{9}$ Department of Primary and Interdisciplinary Care, University of Antwerp, Antwerp, Belgium

\section{Twitter Bernardino Oliva-Fanlo @c_c_baxter}

Acknowledgements The authors would like to thank to the members of the Majorcan Primary Care Research Department and the COGITA group (http://www. gutfeelings.eu) for their helpful advice on the methods used in this protocol.

Contributors BO-F, ME, SM and ES conceived the study, participated in its design and coordination, and helped to draft the manuscript. MM-R, DM and GT participated in the design and helped to draft the manuscript. All authors read and approved the final manuscript.

Funding This work was supported by grants from the Balearic Cancer League (AECC-Baleares, 2017) and the Institute of Health Research Carlos III (PI18/01492). In addition, the corresponding author received an 'Isabel Fernandez' PhD grant in 2016 from the Spanish Society of Family and Community Medicine (semFYC).

Competing interests This study is part of the corresponding author's PhD project. Patient consent for publication Not required.

Provenance and peer review Not commissioned; externally peer reviewed.

Open access This is an open access article distributed in accordance with the Creative Commons Attribution Non Commercial (CC BY-NC 4.0) license, which permits others to distribute, remix, adapt, build upon this work non-commercially, and license their derivative works on different terms, provided the original work is properly cited, appropriate credit is given, any changes made indicated, and the use is non-commercial. See: http://creativecommons.org/licenses/by-nc/4.0/.

ORCID iD

Bernardino Oliva-Fanlo http://orcid.org/0000-0001-7861-1168

\section{REFERENCES}

1 Rubin G, Berendsen A, Crawford SM, et al. The expanding role of primary care in cancer control. Lancet Oncol 2015;16:1231-72.

2 Shapley M, Mansell G, Jordan JL, et al. Positive predictive values of $\geq 5 \%$ in primary care for cancer: systematic review. $\mathrm{Br} \mathrm{J}$ Gen Pract 2010;60:366-77.

3 Walter FM, Rubin G, Bankhead C, et al. Symptoms and other factors associated with time to diagnosis and stage of lung cancer: a prospective cohort study. Br J Cancer 2015;112:S6-13.
4 Johansen M-L, Holtedahl KA, Rudebeck CE. How does the thought of cancer arise in a general practice consultation? Interviews with GPs. Scand J Prim Health Care 2012;30:135-40.

5 Hull FM. The consultation process. In: Sheldon M, Brooke J, Rector A, eds. Decision making in general practice. London: MacMillan, 1985: 13-26.

6 Stolper E, van Bokhoven M, Houben P, et al. The diagnostic role of gut feelings in general practice a focus group study of the concept and its determinants. BMC Fam Pract 2009;10:17.

7 Stolper E, Van Royen P, Van de Wiel M, et al. Consensus on gut feelings in general practice. BMC Fam Pract 2009;10:66.

8 Le Reste J-Y, Coppens M, Barais M, et al. The transculturality of 'gut feelings'. Results from a French Delphi consensus survey. Eur J Gen Pract 2013;19:237-43.

9 Oliva B, March S, Gadea C, et al. Gut feelings in the diagnostic process of Spanish GPs: a focus group study. BMJ Open 2016;6:e012847.

10 Stolper CF, Van de Wiel MWJ, De Vet HCW, et al. Family physicians' diagnostic gut feelings are measurable: construct validation of a questionnaire. BMC Fam Pract 2013;14:1.

11 Barais $\mathrm{M}$, Hauswaldt $\mathrm{J}$, Hausmann $\mathrm{D}$, et al. The linguistic validation of the gut feelings questionnaire in three European languages. BMC Fam Pract 2017:18:54

12 Oliva-Fanlo B, March S, Stolper E, et al. Cross-cultural translation and validation of the ' gut feelings ' questionnaire into Spanish and Catalan. Eur J Gen Pract 2018;0:1-5.

13 Green T, Atkin K, Macleod U. Cancer detection in primary care: insights from general practitioners. Br J Cancer 2015;112:S41-9.

14 Hjertholm P, Moth G, Ingeman ML, et al. Predictive values of GPs' suspicion of serious disease: a population-based follow-up study. $\mathrm{Br}$ $J$ Gen Pract 2014:64:e346-53.

15 Ingeman ML, Christensen MB, Bro F, et al. The Danish cancer pathway for patients with serious non-specific symptoms and signs of cancer-a cross-sectional study of patient characteristics and cancer probability. BMC Cancer 2015;15:421-31.

16 Donker GA, Wiersma E, van der Hoek L, et al. Determinants of general practitioner's cancer-related gut feelings-a prospective cohort study. BMJ Open 2016;6:e012511.

17 Ingebrigtsen SG, Scheel BI, Hart B, et al. Frequency of 'warning signs of cancer' in Norwegian general practice, with prospective recording of subsequent cancer. Fam Pract 2013;30:153-60.

18 Martínez-Cañavate López-Montes T. MODELO MULTINIVEL EXPLICATIVO de la UTILIZACIÓN de las CONSULTAS de ATENCIÓN PRIMARIA en ANDALUCÍA, 2007. Available: http://hera.ugr.es/ tesisugr/16905234.pdf

19 Pacini R, Epstein S. The relation of rational and experiential information processing styles to personality, basic beliefs, and the ratio-bias phenomenon. J Pers Soc Psychol 1999;76:972-87.

20 Witteman C, van den Bercken J, Claes L, et al. Assessing rational and intuitive thinking styles. Eur J Psychol Assess 2009;25:39-47.

21 Chiesa M, Hobbs S. Making sense of social research: how useful is the Hawthorne effect? Eur J Soc Psychol 2008;38:67-74. 